\title{
Bridging the Gap: Towards an Adaptive Video Streaming Approach Supporting Transitions
}

\author{
Julius Rückert^ and David Hausheer \\ Peer-to-Peer Systems Engineering, TU Darmstadt, Germany \\ \{rueckert, hausheer\}@ps.tu-darmstadt.de \\ http://www.ps.tu-darmstadt.de
}

\begin{abstract}
Video streaming over the Internet is becoming increasingly popular. Existing streaming systems range from traditional client/server to peer-to-peer-based approaches. However, no single streaming mechanism fits all possible video streaming scenarios. Therefore, this paper proposes a novel adaptive video streaming approach supporting transitions between different mechanisms in order to provide a stable Quality of Service to streaming clients. Two stages of adaptation are identified and challenges of different transitions are discussed.
\end{abstract}

Keywords: Video Streaming, Transitions, Quality of Service.

\section{Introduction}

Video Streaming over the Internet has increased tremendously and is forecast to gain even more importance in the next years [2]. The increasing number of users, the heterogeneity of end-user devices, and the shift to high definition video content is a challenge for streaming providers.

Traditional client/server $(\mathrm{C} / \mathrm{S})$-based systems, like YouTube, have proven to scale well up to a large number of users, but generate immense costs for the provider [3. At the same time, emerging peer-to-peer $(\mathrm{P} 2 \mathrm{P})$ video streaming techniques provide a cost efficient and flexible alternative by leveraging idle resources of clients to disseminate content to others [4]. Thereby, a key goal of any streaming system is to provide a stable Quality of Service (QoS) to its clients. However, no single mechanism is able to achieve this for all possible video streaming scenarios. Depending on the current conditions (e.g., the number of clients, their resource demand, or the level of dynamics in the system) and the requirements implied by a scenario, different mechanisms are superior over others. For example, while for P2P streaming a tree-based approach performs better in stable conditions, a mesh-based approach shows advantages in case of a high churn rate in dynamic environments. In order to sustain the delivered service quality, it is critical that a video streaming system is able to adapt to such conditions which are not static but may change over time. While adaptations can happen, to some extent, by dynamically changing a mechanism's configuration,

\footnotetext{
* This work has been supported in parts by the German BMBF (\#01BK0806, G-Lab) and by the German DFG (Research Group 733, QuaP2P).
} 
in some cases a switch (herein referred to as transition) to an other streaming mechanism becomes necessary in order to sustain the service quality.

The aim of this paper is to propose a novel video streaming approach that enables transitions between different mechanisms to provide a stable QoS to clients. The support of such transitions can help to realize cost-efficient streaming systems as it overcomes the traditional approach of sticking to a single solution and addressing dynamics in the system only by means of over-provisioning of resources. Thereby, the design of appropriate transition mechanisms in streaming systems is very challenging. For example, a transition mechanism has to support a smooth switching between two approaches without breaking the streaming process of the clients. Moreover, alternative solutions have to be evaluated and compared under different conditions in order to identify valuable transitions. This paper presents two of them and discusses how they relate to each other.

The remainder of this paper is structured as follows. The ideas for an adaptive video streaming system are presented in Section 2 including the description of concrete transitions to be investigated. Subsequently, first results are presented in Section 3. The paper is concluded in Section 4.

\section{Adaptation in Video Streaming}

Starting from a centralized streaming mechanism two major stages of adaptation can be identified, which are detailed in the following. The first stage is defined by the transition between a $\mathrm{C} / \mathrm{S}$ and a $\mathrm{P} 2 \mathrm{P}$-based streaming mechanism. For the $\mathrm{C} / \mathrm{S}$-based streaming, the server, which is the central entity of the system, can be a single server, a server farm, or a service hosted in the cloud that the streaming clients are connected to. This stage of transition can be combined with approaches that aim at adapting central server resources to the needs of the system [6]. The second stage covers the transition between different P2P streaming overlay configurations. Potential configurations include tree-, multitree-, and mesh-based structures, which greatly differ in multiple aspects [5]. For both stages of adaptation, transitions between approaches should not be understood as discrete actions that affects all clients of the system at once. Rather, a transition may initially affect only parts of the system and can be executed gradually up to the entire system.

Transition between C/S- and P2P-Based Streaming. This transition can take place in both directions and can include intermediate steps such as a hybrid solution, where only subsets of the clients adopt a P2P streaming mechanism, whereas others still stream directly from the server. To guarantee a smooth transition, without causing major quality degradations for the clients, an iterative approach is the key. Based on the peers' behavior and their demand for resources, the need for a transition has to be identified, a decision for a target $\mathrm{P} 2 \mathrm{P}$ streaming mechanism has to be taken, and a coordinated transition has to be executed. As long as all clients are directly connected to a common central instance, this entity can serve as coordinator for the transition. It can directly 
inform clients about initial neighbors in the new overlay and, after a certain time, make sure that the clients took part in the transition. In case they did not take part, it can reduce the provided service quality for those clients, making the transition more attractive to them. For the transition in the opposite direction, i.e. from $\mathrm{P} 2 \mathrm{P}$ to $\mathrm{C} / \mathrm{S}$, the tracker, which exists for most streaming overlays, can be used to decide on transitions and coordinate them.

Transition between Different P2P Streaming Mechanisms. Once the system, at least partially, performed a transition to a P2P streaming mechanism, the second stage of adaptation becomes relevant. From here on, transitions between alternative P2P overlay configurations can take place to further react on changing conditions in the system. In the context of this stage, transitions can take place, e.g., between tree-, multi-tree-, and mesh-based topologies as well as between pull- and push-based transmission schemes. Again, these transitions may only be executed in parts of the system, thus either different schemes can exist in parallel or even form a hybrid, where, e.g., stable clients establish a highly efficient tree topology, and other, less stable participants connect to the tree clients and form a mesh-based topology. Hybrid solutions as such have been described before, e.g. by Wang et al. [7. They reflect the properties of a streaming system in the phase of a transition and, hence, are a good starting point towards a full support of transitions in this second stage of adaptation.

Challenges for transitions between different P2P-based mechanisms include the modeling of the single approaches and possible means of transitions between them. Based on this, the potentials for transitions have to be derived and the overhead that an execution of these transitions implies, has to be estimated. To decide on transitions, the system state has to be monitored and evaluated. Furthermore, concrete mechanisms for stepwise and smooth transitions between mechanisms have to be developed.

\section{First Results}

In a former work, the authors addressed quality adaptation in P2P-based Videoon-Demand (VoD) streaming systems [1]. By using Scalable Video Coding (SVC), a video codec that allows to retrieve substreams with different video qualities, the quality of the content can dynamically be matched with available device and system resources. In addition to traditional approaches that focus on QoS aspects, in this work, objectively measured Quality of Experience (QoE) properties of the content were considered for adaptation decisions. It could be shown that by making peers favor always layers with a high estimated QoE but not necessarily high bandwidth requirements, the performance of the entire streaming system can be enhanced in terms of playback delays and SVC video quality by up to $20 \%$. At the same time, content providers are able to reduce up to $60 \%$ of their server capacities, compared to the classical QoS-based approach.

These results are a first step towards the support of transitions in streaming systems. The developed system can be used as starting point for the investigation of transitions between different streaming mechanisms. So far, the system 
presented in [1] applies a mesh-/pull-based approach, which can be extended to allow for transitions between this and other mechanisms such as push-based tree or multi-tree approaches. Furthermore, by considering the streaming of SVC videos, the developed mechanisms for controlled quality adaptations can directly be applied to mitigate undesired influences on the QoS and QoE due to temporal resource bottlenecks in the phase of transition.

\section{Preliminary Conclusions and Future Work}

To deal with highly dynamic scenarios, the support for adaptability by means of transitions between alternative streaming mechanisms has been proposed in this paper. Two stages of adaptation were identified and discussed, including the transition between $\mathrm{C} / \mathrm{S}$ and $\mathrm{P} 2 \mathrm{P}$-based streaming mechanisms and the transition between different P2P-based approaches. Furthermore, ideas for concrete transitions were described that are key for investigations in this area. Initial results in the area of quality adaptation in $\mathrm{P} 2 \mathrm{P}$ streaming systems show a promising first step towards the overall goal.

In future work, additional transitions will be investigated, and other relevant factors that have an influence on transitions will be identified, which will guide the design of suitable mechanisms for the planning and coordination of transitions. As a first step, the modeling and simulation of different dissemination schemes as well as possible transitions between them are planned. Later on, prototypical implementations as extensions to an existing video streaming client and testbed evaluations are planned.

\section{References}

1. Abboud, O., Rückert, J., Hausheer, D., Steinmetz, R.: QoE-aware Quality Adaptation in Peer-to-Peer Video-on-Demand. Tech. Rep. PS-TR-2012-01, Peer-to-Peer Systems Engineering, Technische Universität Darmstadt (2012), http://www.ps.tu-darmstadt.de/fileadmin/publications/PS-TR-2012-01.pdf

2. Cisco Systems Inc.: Cisco VNI: Forecast and Methodology 2010-2015 (2011), http://www.cisco.com/en/US/solutions/collateral/ns341/ns525/ns537/ ns705/ns827/white_paper_c11-481360.pdf

(accessed November 12, 2011)

3. Huang, C., Li, J., Ross, K.W.: Can Internet Video-on-Demand be Profitable? In: ACM SIGCOMM (2007)

4. Liu, Y., Guo, Y., Liang, C.: A Survey on Peer-to-Peer Video Streaming Systems. Peer-to-Peer Networking and Applications 1, 18-28 (2008)

5. Magharei, N., Rejaie, R., Guo, Y.: Mesh or Multiple-tree: A Comparative Study of Live P2P Streaming Approaches. In: IEEE INFOCOM (2007)

6. Pussep, K., Abboud, O., Gerlach, F., Steinmetz, R., Strufe, T.: Adaptive Server Allocation for Peer-assisted Video-on-Demand. In: IEEE IPDPSW, pp. 1-8 (2010)

7. Wang, F., Xiong, Y., Liu, J.: mTreebone: A Hybrid Tree/Mesh Overlay for Application-Layer Live Video Multicast. In: IEEE ICDCS (2007) 